\title{
Prognostic value of magnetic resonance imaging-detected cranial nerve invasion in nasopharyngeal carcinoma
}

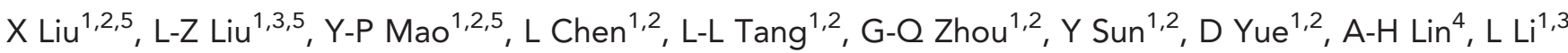
and $\mathrm{JMa}^{*}, 1,2$

${ }^{1}$ State Key Laboratory of Oncology in South China, Collaborative Innovation Center for Cancer Medicine, Sun Yat-sen University Cancer Center, No. 651 Dongfeng Road East, Guangzhou 510060, People's Republic of China; ${ }^{2}$ Department of Radiation Oncology, Sun Yat-sen University Cancer Center, No. 651 Dongfeng Road East, Guangzhou 510060, People's Republic of China; ${ }^{3}$ Imaging Diagnosis and Interventional Center, Sun Yat-sen University Cancer Center, No. 651 Dongfeng Road East, Guangzhou 510060, People's Republic of China and ${ }^{4}$ Department of Medical Statistics and Epidemiology, School of Public Health, Sun Yat-sen University, No. 74 Zhongshan Road, Guangzhou 510060, People's Republic of China

Background: We previously reported that magnetic resonance imaging evidence of cranial nerve invasion was an unfavourable prognostic factor in nasopharyngeal carcinoma. However, the prognostic value of this evidence in nasopharyngeal carcinoma treated with intensity-modulated radiotherapy remains unknown.

Methods: We retrospectively analysed 749 nasopharyngeal carcinoma patients who underwent intensity-modulated radiotherapy.

Results: Cranial nerve invasion was observed in 299 (39.9\%) patients with T3-4 disease. In T3-4 nasopharyngeal carcinoma, magnetic resonance imaging-detected cranial nerve invasion was associated with inferior 5-year overall survival, distant metastasis-free survival, and locoregional relapse-free survival $(P=0.002,0.003$, and 0.012 , respectively). Multivariate analyses confirmed that cranial nerve invasion was an independent prognostic factor for distant metastasis-free survival (hazard ratio, 1.927; $P=0.019$ ) and locoregional relapse-free survival (hazard ratio, 2.605; $P=0.032$ ). Furthermore, the receiver-operating characteristic curves verified that the predictive validity of $T$ classifications was significantly improved when combined with magnetic resonance imaging-detected cranial nerve invasion in terms of death, distant metastasis, and locoregional recurrence $(P=0.015,0.021$ and 0.008 , respectively).

Conclusions: Magnetic resonance imaging-detected cranial nerve invasion is an independent adverse prognostic factor in nasopharyngeal carcinoma treated with intensity-modulated radiotherapy.

Nasopharyngeal carcinoma (NPC) is the most common head and neck $(\mathrm{H} \& \mathrm{~N})$ cancer in south China, with a yearly incidence rate varying between 15 and 50 cases per 100000 (Jemal et al, 2011). Radiotherapy is the primary treatment modality for nondisseminated NPC (Lee et al, 2012). Currently, the Tumour, Node, and Metastasis (TNM) staging system is the most reliable method for determining treatment strategies and predicting outcome.
Moreover, some other clinical and biological features, such as prevertebral space involvement (Zhou et al, 2012), tumour volume (Guo et al, 2012), and Epstein-Barr virus DNA in serum (Lo et al, 2000), have been identified as having important value in risk segregation and survival prediction.

In recent years, diagnostic and treatment methods for NPC have undergone tremendous changes. Intensity-modulated radiation

${ }^{*}$ Correspondence: Professor J Ma; E-mail: majun2@mail.sysu.edu.cn
${ }^{5}$ These authors contributed equally to this work.

Received 10 November 2013; revised 25 December 2013; accepted 8 January 2014; published online 4 February 2014

(c) 2014 Cancer Research UK. All rights reserved 0007-0920/14 
therapy (IMRT) in particular has gradually replaced two-dimensional conventional radiotherapy (2D-CRT) as the primary radiotherapy technique based on improved tumour target conformity, as well as superior locoregional control and long-term survival in NPC (Lee et al, 2002; Kam et al, 2004). Therefore, it is of great importance to determine whether the prognostic factors previously identified in patients treated with 2D-CRT can also be applied in the IMRT era.

Perineural invasion (PNI) is a key clinicopathological feature and established adverse prognostic factor in many malignancies, including $\mathrm{H} \& \mathrm{~N}$, colon and rectum, and prostate cancers (Bernier et al, 2004; Harnden et al, 2007; Liebig et al, 2009). Similarly, cranial nerve $(\mathrm{CN})$ involvement has been proven to be a poor prognostic factor in NPC (Chang et al, 2005). According to the 7th Union for International Cancer Control/American Joint Committee on Cancer (UICC/AJCC) staging system, NPC with CN involvement are classified as T4 (Edge et al, 2010). However, because surgery is not an option in NPC, except as a salvage treatment, $\mathrm{CN}$ involvement is assessed by neurological evaluation, which may underestimate the incidence and importance of this key feature of tumour behaviour (Su and Lui, 1996; Liu et al, 2009).

Magnetic resonance imaging, which has excellent soft-tissue contrast resolution and multiplanar imaging capability, has been proven to be a valuable tool for detecting and defining the extent of $\mathrm{CN}$ invasion in H\&N cancers, including NPC (Liu et al, 2009; Ong and Chong, 2010). We have previously reported that MRI-detected $\mathrm{CN}$ invasion had an unfavourable impact on the prognosis of NPC (Liu et al, 2009). However, the prognostic role of MRI-detected CN invasion in NPC treated with IMRT remains unknown. Thus, the aim of the present study was to determine the prognostic value of MRI-detected CN invasion in a large cohort of NPC patients treated with IMRT.

\section{MATERIALS AND METHODS}

Patient characteristics. We retrospectively reviewed the records of all 749 newly diagnosed, biopsy-proven, non-metastatic NPC patients treated using IMRT at Sun Yat-sen University Cancer Center between January 2003 and December 2007. The clinical features of these patients are shown in Table 1.

All patients completed a pretreatment evaluation including complete patient history, physical examination, hematology and biochemistry profiles, nasopharynx and neck MRI, chest radiography, abdominal ultrasonography, and single photon emission computed tomography (SPECT) whole-body bone scan. Positron emission tomography computed tomography (PET-CT) was performed in 162 of 749 patients (21.6\%). All patients were restaged according to the 7 th UICC/AJCC staging system. This retrospective study was conducted in compliance with the institutional policy to protect the patients' private information and was approved by the institutional review board of Sun Yat-Sen University Cancer Center. Informed consent was obtained from all patients.

Imaging protocol. All patients underwent MRI with a $1.5-\mathrm{T}$ system (General Electric Signa CV/i; General Electric Healthcare, Chalfont St Giles, UK). The region from the suprasellar cistern to the inferior margin of the sternal end of the clavicle was examined with an $\mathrm{H} \& \mathrm{~N}$ combined coil. T1-weighted fast spin-echo images in the axial, coronal, and sagittal planes (repetition time, 500-600 ms; echo time, 10-20 ms) and T2-weighted fast spin-echo images in the axial plane (repetition time, $4000-6000 \mathrm{~ms}$; echo time, 95-110 ms) were obtained before injecting the contrast material. After intravenous administration of gadopentetate dimeglumine (GdDTPA; Magnevist, Schering, Berlin) at a dose of $0.1 \mathrm{mmol}$ per $\mathrm{kg}$ body weight, spin-echo T1-weighted axial and sagittal sequences
Table 1. Clinical features of the 749 NPC patients in the study Characteristic No. of patients (\%)

\begin{tabular}{|l|l|}
\hline \multicolumn{2}{|l|}{ Age (years) } \\
\hline$>50$ & $553(73.8)$ \\
$\leqslant 50$ & $196(26.2)$
\end{tabular}

\section{Gender}

\begin{tabular}{|l|l|}
\hline Male & $580(77.4)$ \\
Female & $169(22.6)$
\end{tabular}

\section{WHO pathology}

\begin{tabular}{|l|r}
\hline Type I & $5(0.7)$ \\
Type II/III & $744(99.3)$ \\
\hline
\end{tabular}

\section{Chemotherapy}

Concurrent chemoradiotherapy $535(71.4)$

Neoadjuvant + concurrent chemoradiotherapy Concurrent chemoradiotherapy + adjuvant $233(31.1)$

$246(32.8)$

$46(8.6)$

$214(28.6)$

\begin{tabular}{|l|l|}
\hline T category $^{\text {a }}$ \\
\hline T1 & $177(23.6)$ \\
T2 & $140(18.7)$ \\
T3 & $264(35.2)$ \\
T4 & $168(22.4)$
\end{tabular}

$\mathrm{N}$ category $^{\mathrm{a}}$

\begin{tabular}{|c|c|}
\hline N0 & $184(24.6)$ \\
N1 & $409(54.6)$ \\
N2 & $106(14.2)$ \\
N3 & $50(6.7)$ \\
\hline
\end{tabular}

\section{Stage $\mathrm{a}^{\mathrm{a}}$} I II III IV $210(28.0)$

Abbreviations: $\mathrm{AJCC}=$ American Joint Committee on Cancer; $\mathrm{N}=$ node; $\mathrm{NPC}=$ nasopharyngeal carcinoma; $\mathrm{T}=$ tumour; $\mathrm{UICC}=$ Union for International Cancer Control; $W H O=$ World Health Organization

${ }^{\text {a }}$ According to the 7 th UICC/AJCC staging system.

and spin-echo T1-weighted fat-suppressed coronal sequences were performed sequentially, with parameters similar to those used before Gd-DTPA injection. We used a section thickness of $3 \mathrm{~mm}$ for coronal sequences and $5 \mathrm{~mm}$ for other sequences; 22-cm field of view; and $320 \times 224$ matrix.

Image assessment. Two radiologists specializing in $\mathrm{H} \& \mathrm{~N}$ cancers, who were blinded to the clinical findings, evaluated the MRI data separately. Any disagreements were resolved by consensus. Cranial nerve invasion was detected on images that met one of the following criteria: (1) enhancing soft-tissue tumour along the course of the ipsilateral-related nerve, replacing the normal structures of the $\mathrm{CN}$ on gadolinium-enhanced T1-weighted images; and (2) perineural spread, defined as an enlargement or abnormal enhancement of the nerve, obliteration of the neural fat pads adjacent to the neurovascular foramina, and neuroforaminal enlargement (Figure 1) (Liu et al, 2009; Ong and Chong, 2010). Because $\mathrm{CNs}$ in the parapharyngeal space could not be identified as isolated structures on MRI, parapharyngeal space invasion could not be regarded as evidence of $\mathrm{CN}$ invasion in our study. 

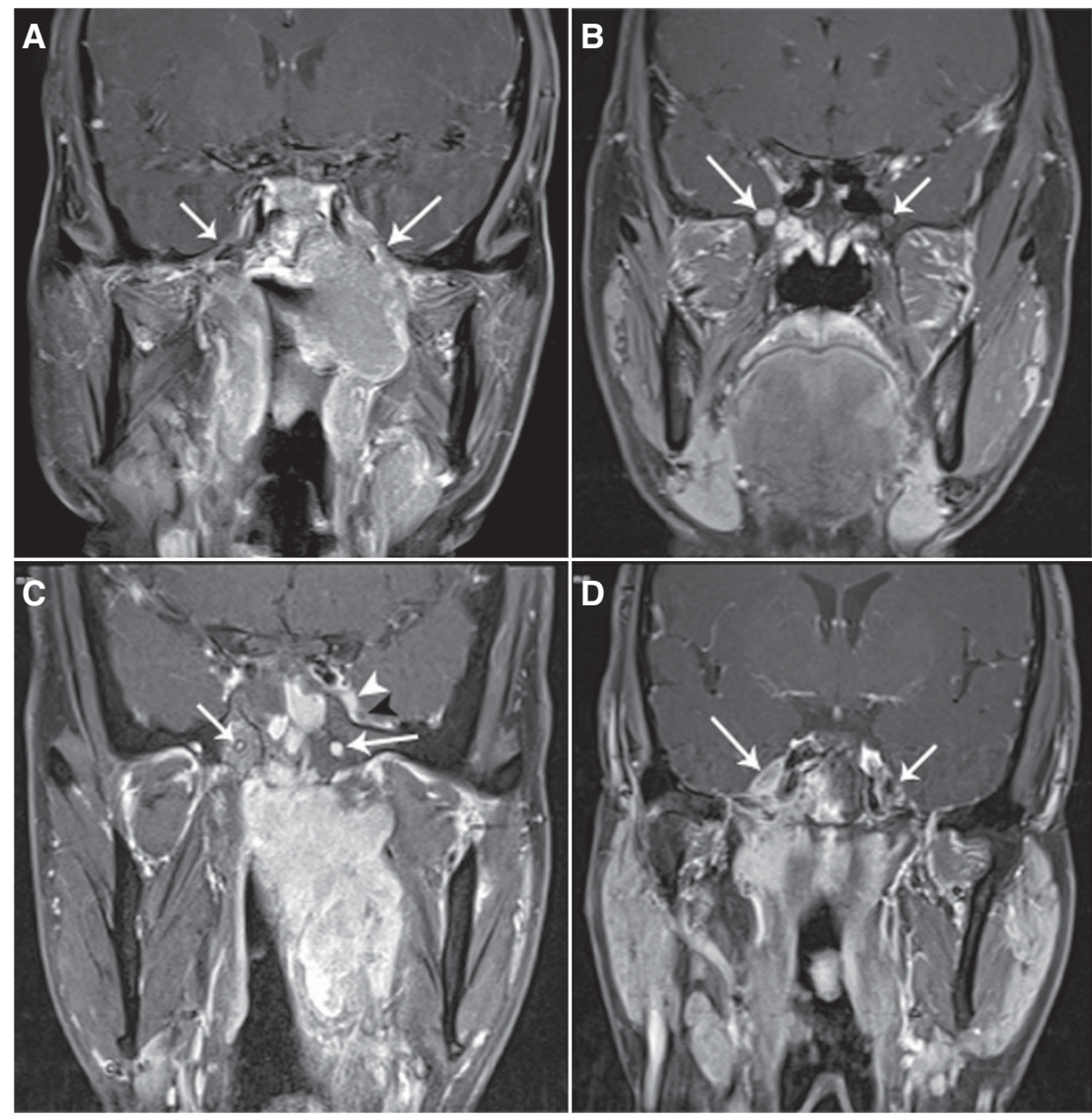

Figure 1. Enhanced, coronal, T1-weighted, fat-suppression magnetic resonance (MR) images (repetition time, $600 \mathrm{~ms}$; echo, $15 \mathrm{~ms}$ ) of patients with nasopharyngeal carcinoma. (A) A patient with left CN V3 palsy. Image shows perineural invasion via the enlarged right foramen ovale along V3 (long arrow) and into the cavernous sinus. The main branch of the V3 on the right is normal (short arrow). (B) A patient with right CN V2 palsy. Image shows enlargement of the right foramen rotundum and enhancement of the right V2 (long arrow). The left foramen rotundum and V2 is normal (short arrow). (C) A patient with left CN V1 palsy. Image shows abnormal enhancement of the left V1 (white arrowhead) and enlargement and enhancement of the left pterygoid canal (long arrow). Note that the left V2 (black arrowhead) and the right pterygoid canal (short arrow) is not involved. (D) A patient without clinical CN palsy. Image shows abnormal enhancement of the right Gasser's ganglion (long arrow). The left Gasser's ganglion is not involved (short arrow).

Magnetic resonance imaging findings of $\mathrm{CN}$ invasion at the following sites were assessed: extracranial segment of the maxillary nerve (V3), pterygopalatine fossa, foramen rotundum, foramen ovale, pterygoid canal, internal acoustic meatus, jugular foramen, hypoglossal canal, inferior orbital fissure, orbital apex, superior orbital fissure, cavernous sinus segment of CNs III-V2 and VI, trigeminal ganglion, and $\mathrm{CNs}$ in the cistern.

Treatment. All patients underwent radical radiation therapy. The nasopharyngeal and upper neck tumour volumes were treated using IMRT for the entire treatment course. The lower neck was treated with a conventional anterior or anteroposterior opposing cervical technique. Further details of the IMRT techniques used at Sun Yat-Sen University Cancer Center have been reported previously (Lai et al, 2011).

During the study period, our institutional guidelines recommended no chemotherapy for stage I to IIA, concurrent chemoradiation therapy for stage IIB, and concurrent chemoradiation therapy with or without neoadjuvant/adjuvant chemotherapy for stage III to IVA-B, as defined by the 6th edition of the UICC/ AJCC staging system. In total, $86.2 \%$ of stage III and IV patients (424 of 492) received chemotherapy. When possible, salvage treatments, such as intracavitary brachytherapy, surgery, and chemotherapy, were provided in the event of documented relapse or persistent disease.
Follow-up. The median follow-up was 81.4 months (range, 3.1-126.5 months). Each patient was assessed for treatment response and toxicity during (weekly) and after radiotherapy (every 2-3 months during the first 2 years; every 3-6 months during years 3-5). Endoscopy, CT, or MRI scans of the head and neck were performed every 3 months during the first year and annually during years 2-5. Patients with residual or recurrent local disease underwent biopsy to confirm malignancy. Additional tests were ordered when indicated to evaluate for local or distant failure.

Statistical analysis. All analyses were performed using SPSS version 20.0 (IBM Corporation, Armonk, NY, USA). We compared the categorical variables in different groups by using the $\chi^{2}$ test (or Fisher's exact test, if indicated). Actuarial rates were estimated by the Kaplan-Meier method; survival curves were compared using the log-rank test (Kaplan and Meier, 1958). The following end points were estimated: overall survival (OS), distant metastasis-free survival (DMFS), and locoregional relapse-free survival (LRFS). We calculated OS from the first day of treatment to death. For DMFS and LRFS analyses, we recorded the latencies (i.e., time from the first day of treatment) to the first distant and locoregional failure.

Multivariate analyses with the Cox proportional hazards model were used to test for independent significance by backward elimination of insignificant explanatory variables (Cox, 1972). 
The Cox proportional hazards model was also used to calculate the hazard ratio (HR). The area under the receiver-operating characteristic (ROC) curve was used to assess the predictive validity of MRI-detected $\mathrm{CN}$ invasion, based on the method described by Hanley and McNeil (1982) and Zweig and Campbell (1993). The criterion for statistical significance was set at $\alpha=0.05$. All $P$-values were based on two-sided tests.

\section{RESULTS}

Clinical diagnosis of $\mathrm{CN}$ palsies and MRI findings of $\mathrm{CN}$ invasion. Of the total 749 patients, 72 (9.8\%) patients initially presented with CN palsy. A total of $188 \mathrm{CNs}$ (the three divisions of trigeminal nerve were counted separately) were found to be paralysed, and 32 patients were found to have multiple $\mathrm{CN}$ palsies. Of the 72 patients with $\mathrm{CN}$ palsy, 71 patients had one or more MRI findings demonstrating $\mathrm{CN}$ invasion.

Magnetic resonance imaging evidence of $\mathrm{CN}$ invasion was found in 299 (39.9\%) patients, all of whom had locally advanced (T3-4) disease. The incidence of MRI-detected CN invasion in T3 patients was significantly lower than in T4 patients $(53.4 \%$ vs $94.0 \%, P<0.001)$. The correlation between MRI-detected $\mathrm{CN}$ invasion and ipsilateral $\mathrm{CN}$ palsy is shown in Table 2.

Imaging basis of CN palsies. Among the 188 paralysed CNs, 163 $(86.7 \%)$ were found to have MRI evidence of CN invasion. No MRI evidence of $\mathrm{CN}$ invasion was observed in the remaining $25(13.3 \%)$ paralysed CNs. In these $25 \mathrm{CNs}$, carotid sheath $(n=2)$ or retropharyngeal lymph node $(n=1)$ invasion could be identified along the course of ipsilaterally paralysed CNs. No tumour could be detected along the course of the remaining paralysed $\mathrm{CNs}$ on MRI.

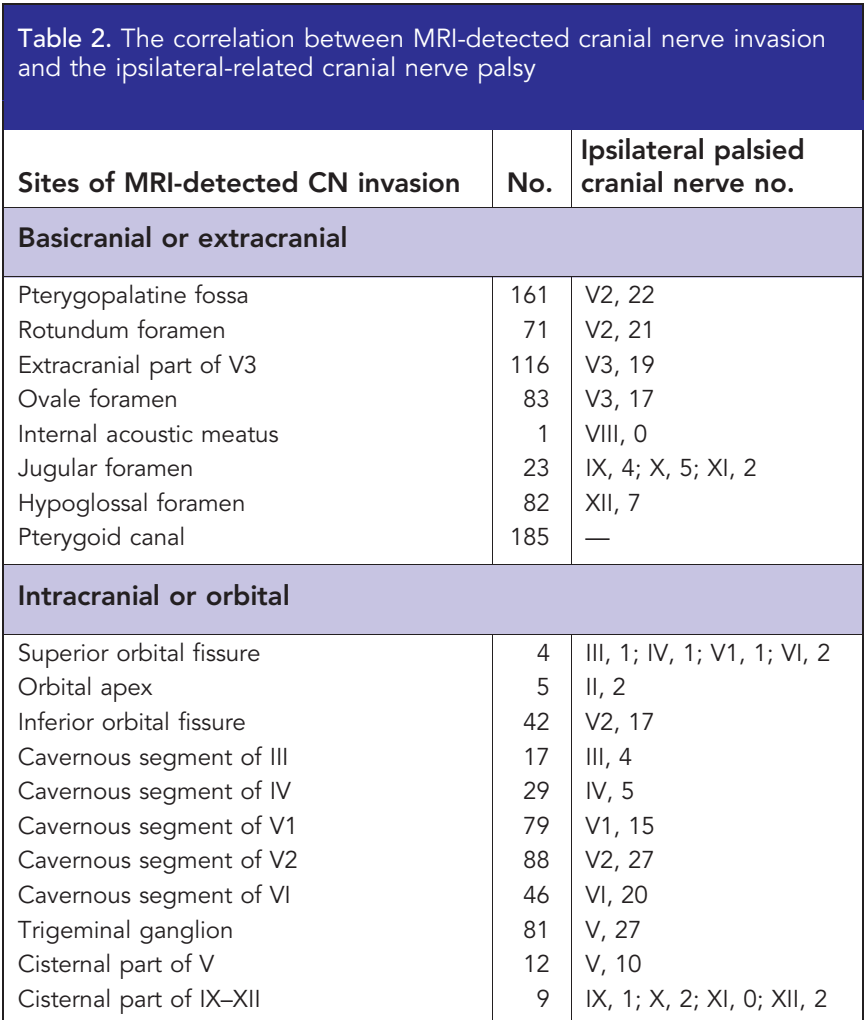

Abbreviations: $\mathrm{CN}=$ cranial nerve; $\mathrm{MRI}=$ magnetic resonance imaging.
Prognostic value of MRI-detected $\mathrm{CN}$ invasion in T3-4 disease. In 432 patients with T3-4 disease, the 5-year OS (75.8\% vs $87.9 \%$, $P=0.002)$, DMFS (73.5\% vs $86.2 \%, P=0.003)$, and LRFS $(88.2 \%$ vs $95.9 \%, P=0.012)$ for patients with MRI-detected CN invasion were significantly lower than the corresponding rates for patients without MRI-detected CN invasion.

Because most T4 patients had MRI evidence of $\mathrm{CN}$ invasion, the prognosis of T3 patients with and without MRI-detected CN invasion was also compared. All patients with locally advanced disease (T3 and T4) in this series were classified into three groups: group 1, T3 disease without MRI-detected CN invasion; group 2, T3 disease with MRI-detected $\mathrm{CN}$ invasion; and group 3, T4 disease. There was no significant difference between groups 2 and 3 with respect to OS $(80.1 \%$ vs $73.1 \%, P=0.138)$, DMFS $(77.8 \%$ vs $70.8 \%, P=0.345)$, and LRFS ( $87.9 \%$ vs $89.3 \%, P=0.611$; Figure 2$)$. However, patients in group 2 had significantly lower DMFS $(77.8 \%$ vs $85.9 \%, P=0.047)$ and LRFS $(87.9 \%$ vs $95.6 \%, P=0.025)$ than patients in group 1 , and trended towards inferior OS $(80.1 \%$ vs $87.7 \%, P=0.063$ ) (Figure 2).

Multivariate analysis was performed to adjust for various prognostic factors in T3 and T4 disease. The following parameters were included in the Cox proportional hazards model by backward elimination of insignificant explanatory variables: age $(\leqslant 50$ years $v s>50$ years), gender, WHO pathology type (I vs II amd III), skull base invasion, paranasal sinus involvement, intracranial invasion, infratemporal invasion, orbital invasion, hypopharynx involvement, MRI-detected CN invasion, $\mathrm{CN}$ palsy, $\mathrm{N}$ classification, and chemotherapy. MRI-detected $\mathrm{CN}$ invasion was found to be an independent predictive factor for DMFS (HR, 1.927; 95\% CI, $1.116-3.327 ; P=0.019)$ and LRFS (HR, 2.605; 95\% CI, $1.085-$ $6.255 ; P=0.032$ ) rates in locally advanced disease (Table 3 ). Patients with MRI-detected CN invasion also had an increased risk of death, this difference was marginally significant (HR, 1.592; 95\% CI, 0.942-2.689; $P=0.082$; Table 3).

Difference in the prognostic implications between clinical $\mathrm{CN}$ palsy and MRI-detected $\mathrm{CN}$ invasion. In the 300 patients with clinical or MRI-detected CN invasion, no significant difference was observed in 5-year DMFS (70.7\% vs $74.4 \%, P=0.662)$ or LRFS (85.4\% vs $89.2 \%, P=0.289$ ) between patients with and without clinical CN palsy. A marginally significant difference was observed in the 5 -year OS $(70.8 \%$ vs $77.5 \%, P=0.053)$ rate.

The prognostic validity of adding MRI-detected $\mathrm{CN}$ invasion to $T$ classification in NPC. Receiver-operating characteristic curves were used to evaluate the prognostic validity of adding MRIdetected $\mathrm{CN}$ invasion to the $\mathrm{T}$ classification of TNM staging system. In the 749 patients with NPC, the AUC significantly increased when MRI-detected $\mathrm{CN}$ invasion was added to the $\mathrm{T}$ classification regarding death $(0.663$ vs $0.651, P=0.015$; Figure $3 \mathrm{~A}), \mathrm{DM}(0.661$ vs $0.648, P=0.021$; Figure $3 \mathrm{~B})$, and locoregional recurrence ( 0.638 vs $0.619, P=0.008$; Figure $3 C$ ). These results reveal that, in predicting outcomes, the combination of MRI-detected $\mathrm{CN}$ invasion and $\mathrm{T}$ classification was superior to $\mathrm{T}$ classification alone. To classify NPC with cranial nerve involvement (clinical palsy or MRI-detected invasion) as T4 warrants further consideration in the future revision of the current TNM staging system.

\section{DISCUSSION}

Perineural invasion has been described in the literature since the mid-1800s but has received relatively little research attention (Liebig et al, 2009). In H\&N cancers, PNI is a well-recognised prognostic factor and an important indication for adjuvant chemoradiotherapy or radiotherapy after surgery (Bernier et al, 2004). Cranial nerve palsy is common in locally advanced NPC, 
A

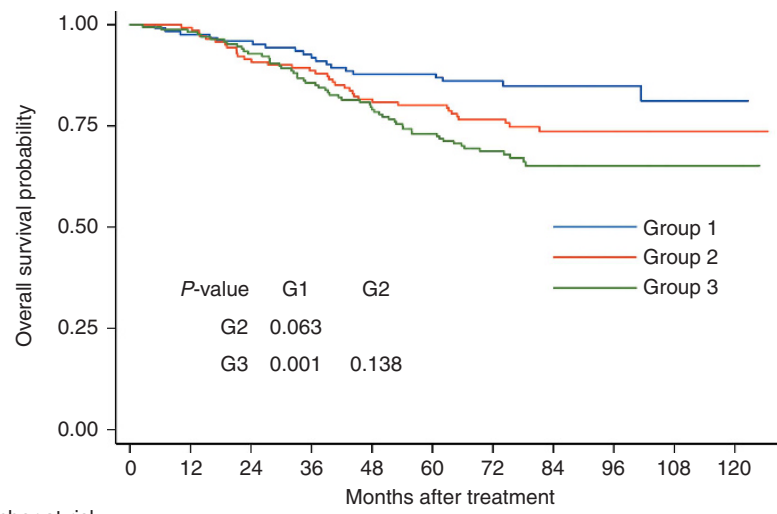

Number at risk

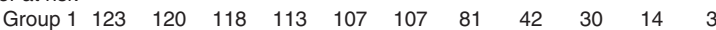
$\begin{array}{llllllllllll}\text { Group } 2 & 141 & 140 & 129 & 125 & 115 & 113 & 91 & 55 & 32 & 17 & 4\end{array}$

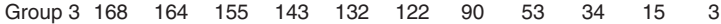

B

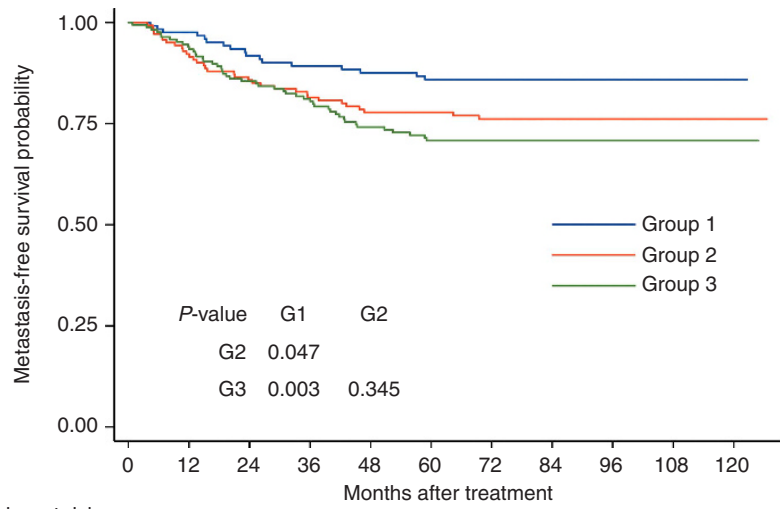

Number at risk

$\begin{array}{llllllllllll}\text { Group } 1 & 123 & 118 & 111 & 106 & 104 & 102 & 78 & 39 & 28 & 13 & 3\end{array}$

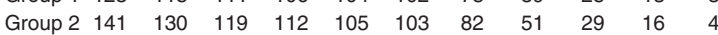

$\begin{array}{llllllllllll}\text { Group } 3 & 168 & 156 & 138 & 128 & 115 & 105 & 79 & 51 & 32 & 14 & 3\end{array}$

C

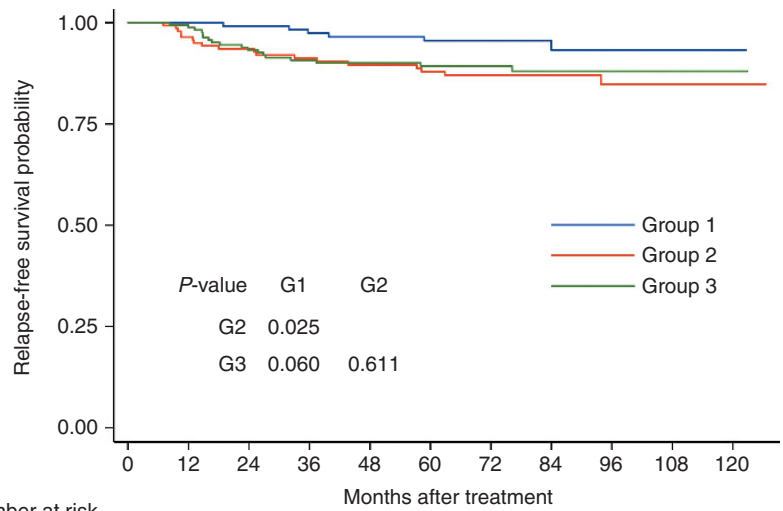

Number at risk

$\begin{array}{llllllllllll}\text { Group } 1 & 123 & 120 & 117 & 110 & 104 & 103 & 79 & 40 & 28 & 13 & 3\end{array}$

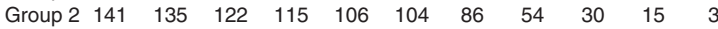

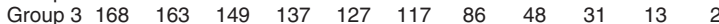

Figure 2. Kaplan-Meier survival curves for overall survival (A), distant metastasis-free survival (B), and locoregional relapse-free survival (C) in different groups: group 1, T3 disease without MRI-detected $\mathrm{CN}$ invasion; group 2, T3 disease with MRl-detected CN invasion; group 3, T4 disease. P-values were calculated with the unadjusted log-rank test. Abbreviations: $\mathrm{CN}=$ Cranial nerve; $\mathrm{MRI}=$ Magnetic resource imaging.

which clearly indicates the existence of neural invasion. However, PNI is often insidious and asymptomatic, especially in the early stages (Su and Lui, 1996). It was not until the advent of crosssectional imaging (CT and MRI) that the comprehensive study of PNI in NPC became possible. Consistent with the results of previous studies, our results showed that the incidence of $\mathrm{CN}$

\begin{tabular}{|c|c|c|c|c|}
\hline End point & Variable & $P$-value ${ }^{\text {a }}$ & HR & $\begin{array}{l}95 \% \mathrm{Cl} \\
\text { for } \mathrm{HR}\end{array}$ \\
\hline \multirow[t]{5}{*}{ OS } & $\begin{array}{l}\text { MRI-detected CN } \\
\text { invasion }\end{array}$ & 0.082 & 1.592 & $0.942-2.689$ \\
\hline & Age & $<0.001$ & 2.160 & $1.471-3.172$ \\
\hline & Intracranial invasion & 0.022 & 1.630 & $1.072-2.479$ \\
\hline & Orbital invasion & 0.070 & 1.662 & $0.959-2.878$ \\
\hline & $\mathrm{N}$ category $^{\mathbf{b}}$ & 0.043 & 1.557 & $1.014-2.391$ \\
\hline \multirow[t]{3}{*}{ DMFS } & $\begin{array}{l}\text { MRl-detected CN } \\
\text { invasion }\end{array}$ & 0.019 & 1.927 & $1.116-3.327$ \\
\hline & Intracranial invasion & 0.036 & 1.583 & $1.031-2.429$ \\
\hline & $\mathrm{N}$ category $^{\mathbf{b}}$ & 0.001 & 2.060 & $1.346-3.153$ \\
\hline \multirow[t]{2}{*}{ LRFS } & $\begin{array}{l}\text { MRI-detected CN } \\
\text { invasion }\end{array}$ & 0.032 & 2.605 & $1.085-6.255$ \\
\hline & Orbital invasion & 0.075 & 2.113 & $0.928-4.813$ \\
\hline \multicolumn{5}{|c|}{ 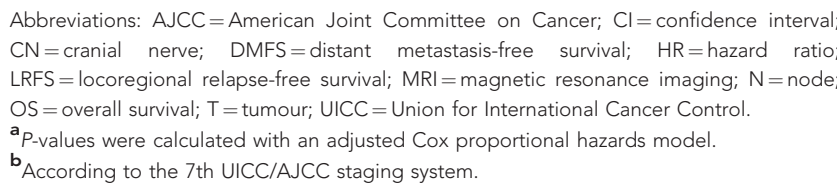 } \\
\hline
\end{tabular}

invasion detected by MRI was much higher than that of clinical CN palsy (Su and Lui, 1996; Liu et al, 2009). Furthermore, we demonstrated that MRI-detected CN invasion, whether symptomatic or asymptomatic, still indicated poorer survival in locally advanced NPC in the IMRT era, and combining MRI-detected CN invasion with the current $\mathrm{T}$ classification resulted in better prediction of death, DM, and locoregional recurrence.

The underlying mechanisms behind PNI are not fully understood. For the past 40 years, the predominant theory behind the pathogenesis of PNI has been that tumour cells spreading along neural sheaths are privileged with low resistance (Liebig et al, 2009). However, recent studies have revealed that PNI may involve active reciprocal signalling interactions between tumour cells and nerves and that these invading tumour cells may have acquired the ability to respond to proinvasive signals within the peripheral nerve milieu (Liebig et al, 2009). In a PNI model using mouse dorsal root ganglia (DRG) cocultured in a Matrigel matrix with prostate cancer cells, Ayala et al (2001)demonstrated tumour cell migration along neurites towards the ganglia of origin as well as focused, directional outgrowth of neurites towards cancer cell colonies. Moreover, the growth of cancer cells and neurite outgrowth of DRG cells was elevated when cultured together (Ayala et al, 2001).

There is a growing body of literature reporting neurotrophic factor involvement in cancer and its association with PNI (Zhu et al, 1999; Liebig et al, 2009). Jimenez-Andrade et al (2011) reported that preventive or late administration of anti-nerve growth factor therapy attenuates tumour-induced nerve sprouting, neuroma formation, and cancer pain in a mouse model of prostate cancer. Bakst et al (2012) demonstrated in vitro and in vivo that radiation impairs PNI through downregulating the expression of glial-derived neurotrophic factor by DRG and inhibiting cancer cell viability. Taken together, these results strongly suggest that PNI is not only just a route of tumour spread but also a marker of aggressive tumour behaviour ( $\mathrm{Zhu}$ et al, 1999). This may explain the propensity of NPC to spread through neural foraminas and the prognostic value of MRI-detected CN invasion in NPC.

As non-disseminated NPC was not treated by surgery, we were not able to correlate pathological examination with MRI-detected 

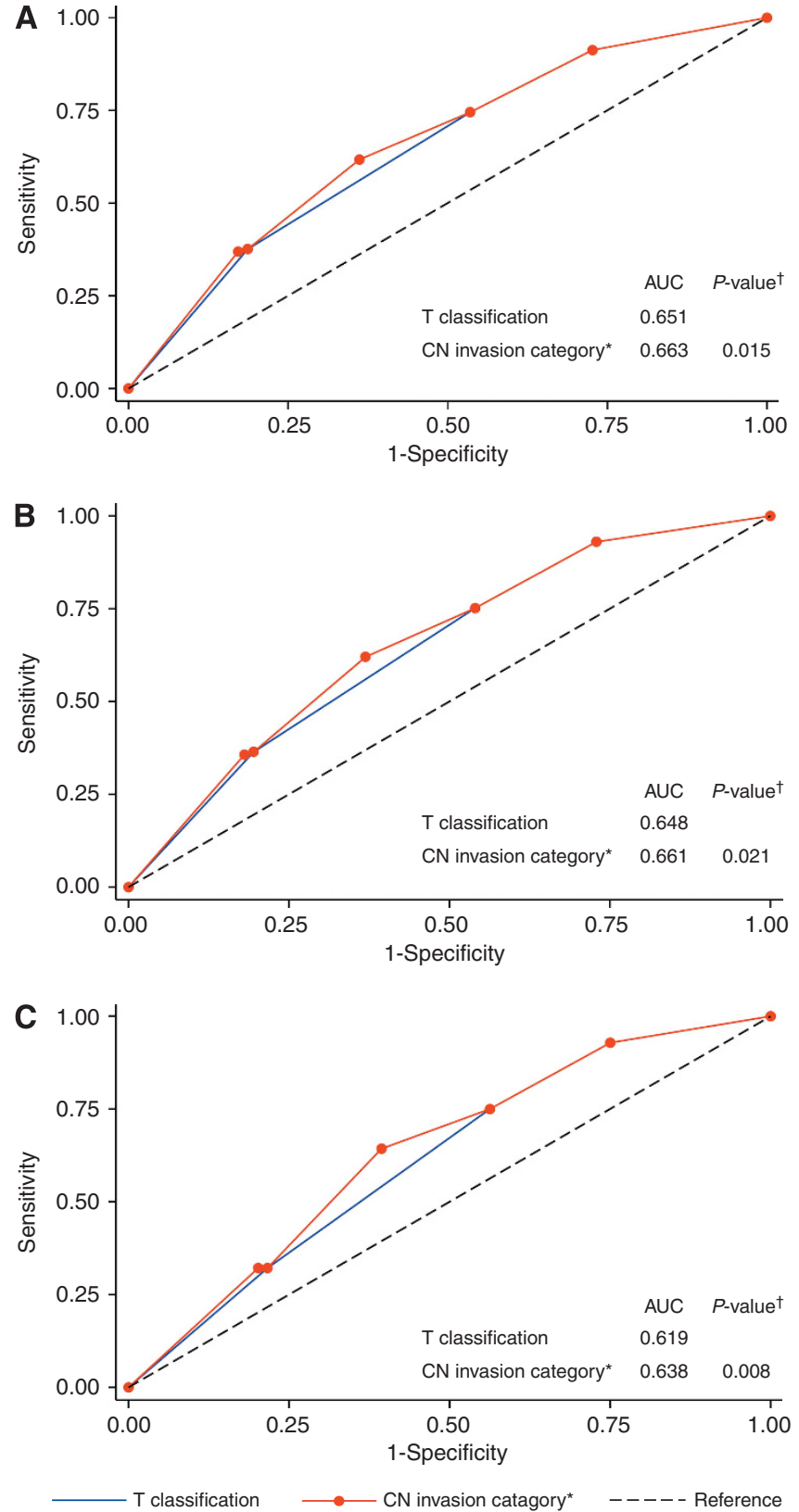

Figure 3. Receiver-operating characteristic curves for the outcome prediction of $\mathrm{T}$ classification alone and the combination of magnetic resonance imaging (MRI)-detected $\mathrm{CN}$ invasion and $\mathrm{T}$ classification in NPC $(n=749)$, with death $(\mathbf{A})$, distant metastasis (B), and locoregional recurrence $(\mathbf{C})$ as the end point. The $P$-values were compared with the $\mathrm{T}$ classification alone. *Magnetic resonance imaging-detected $\mathrm{CN}$ invasion combined with T classification. Abbreviations: $\mathrm{AUC}=$ area under the curve; $\mathrm{CN}=$ Cranial nerve; $\mathrm{MRI}=$ Magnetic resource imaging.

CN invasion, which was the major limitation of our study. However, MRI evidences of $\mathrm{CN}$ invasion along the course of paralysed nerves were found in most cases (86.7\%). Moreover, by comparing the results of MRI with postoperative pathological examination, Hanna et al (2007) proved that MRI had a very satisfactory sensitivity (100\%) and specificity (85\%) for detecting perineural spread of adenoid cystic carcinoma of the $H \& N$ to the skull base, which is comparable to other reports in H\&N cancer (Gandhi et al, 2011).

To the best of our knowledge, herein is the first evidence demonstrating the prognostic value of MRI-detected $\mathrm{CN}$ invasion in NPC treated with IMRT. Our results show that patients with
MRI-detected $\mathrm{CN}$ invasion experienced more recurrences and distant metastases, which suggested that current chemoradiotherapy strategies might not be sufficient for this subset of patients. Further studies are required to develop more intensive systemic approaches combined with IMRT to improve the outcome of these patients. Moreover, researchers have proven that antineurotrophin therapy was effective in breast, prostate, and pancreatic cancer in vivo (Miknyoczki et al, 2002; Adriaenssens et al, 2008). The therapeutic potential of this novel strategy in NPC with CN invasion needs to be addressed in the future.

In conclusion, MRI-detected CN invasion is common in NPC and often seen in asymptomatic patients. MRI-detected CN invasion confers poorer prognoses in NPC patients treated with IMRT. Future studies are warranted to reveal the latent mechanism behind $\mathrm{CN}$ invasion and to develop more effective treatment strategies in NPC patients with MRI-detected CN invasion.

\section{ACKNOWLEDGEMENTS}

This work was supported by grants from the Guangdong Province Universities and Colleges Pearl River Scholar Funded Scheme (2010), the Innovation Team Development Plan of the Ministry of Education (No. IRT1297), the National Natural Science Foundation of China (No. 81230056), and the Sun Yat-sen University Clinical Research 5010 Program (No. 2012011).

\section{CONFLICT OF INTEREST}

The authors declare no conflict of interest.

\section{REFERENCES}

Adriaenssens E, Vanhecke E, Saule P, Mougel A, Page A, Romon R, Nurcombe V, Le Bourhis X, Hondermarck H (2008) Nerve growth factor is a potential therapeutic target in breast cancer. Cancer Res 68: 346-351.

Ayala GE, Wheeler TM, Shine HD, Schmelz M, Frolov A, Chakraborty S, Rowley D (2001) In vitro dorsal root ganglia and human prostate cell line interaction: redefining perineural invasion in prostate cancer. Prostate 49: 213-223.

Bakst RL, Lee N, He S, Chernichenko N, Chen CH, Linkov G, Le HC, Koutcher J, Vakiani E, Wong RJ (2012) Radiation impairs perineural invasion by modulating the nerve microenvironment. PLoS One 7: e39925.

Bernier J, Domenge C, Ozsahin M, Matuszewska K, Lefebvre JL, Greiner RH, Giralt J, Maingon P, Rolland F, Bolla M, Cognetti F, Bourhis J, Kirkpatrick A, van Glabbeke M (2004) Postoperative irradiation with or without concomitant chemotherapy for locally advanced head and neck cancer. N Engl J Med 350: 1945-1952.

Chang JT, Lin CY, Chen TM, Kang CJ, Ng SH, Chen IH, Wang HM, Cheng AJ, Liao CT (2005) Nasopharyngeal carcinoma with cranial nerve palsy: the importance of MRI for radiotherapy. Int J Radiat Oncol Biol Phys 63: 1354-1360.

Cox DR (1972) Regression models and life-tables. J R Stat Soc Ser B (Methodological) 34: 187-220.

Edge SB, Byrd DR, Compton CC, Fritz AG, Greene FL, Trotti A (2010) American Joint Committee on Cancer Cancer Staging Manual. 7th edn, pp 41-56. Springer: New York, NY, USA.

Gandhi MR, Panizza B, Kennedy D (2011) Detecting and defining the anatomic extent of large nerve perineural spread of malignancy: comparing 'targeted' MRI with the histologic findings following surgery. Head Neck 33: 469-475.

Guo R, Sun Y, Yu XL, Yin WJ, Li WF, Chen YY, Mao YP, Liu LZ, Li L, Lin AH, Ma J (2012) Is primary tumor volume still a prognostic factor in intensity modulated radiation therapy for nasopharyngeal carcinoma? Radiother Oncol 104: 294-299.

Hanley JA, McNeil BJ (1982) The meaning and use of the area under a receiver operating characteristic (ROC) curve. Radiology 143: 29-36. 
Hanna E, Vural E, Prokopakis E, Carrau R, Snyderman C, Weissman J (2007) The sensitivity and specificity of high-resolution imaging in evaluating perineural spread of adenoid cystic carcinoma to the skull base. Arch Otolaryngol Head Neck Surg 133: 541-545.

Harnden P, Shelley MD, Clements H, Coles B, Tyndale-Biscoe RS, Naylor B, Mason MD (2007) The prognostic significance of perineural invasion in prostatic cancer biopsies: a systematic review. Cancer 109: 13-24.

Jemal A, Bray F, Center MM, Ferlay J, Ward E, Forman D (2011) Global cancer statistics. CA Cancer J Clin 61: 69-90.

Jimenez-Andrade JM, Ghilardi JR, Castaneda-Corral G, Kuskowski MA, Mantyh PW (2011) Preventive or late administration of anti-NGF therapy attenuates tumor-induced nerve sprouting, neuroma formation, and cancer pain. Pain 152: 2564-2574.

Kam MK, Teo PM, Chau RM, Cheung KY, Choi PH, Kwan WH, Leung SF, Zee B, Chan AT (2004) Treatment of nasopharyngeal carcinoma with intensity-modulated radiotherapy: the Hong Kong experience. Int J Radiat Oncol Biol Phys 60: 1440-1450.

Kaplan EL, Meier P (1958) Nonparametric estimation from incomplete observations. J Am Stat Assoc 53: 457-481.

Lai SZ, Li WF, Chen L, Luo W, Chen YY, Liu LZ, Sun Y, Lin AH, Liu MZ, Ma J (2011) How does intensity-modulated radiotherapy versus conventional two-dimensional radiotherapy influence the treatment results in nasopharyngeal carcinoma patients? Int J Radiat Oncol Biol Phys 80: 661-668.

Lee AW, Ng WT, Chan YH, Sze H, Chan C, Lam TH (2012) The battle against nasopharyngeal cancer. Radiother Oncol 104: 272-278.

Lee N, Xia P, Quivey JM, Sultanem K, Poon I, Akazawa C, Akazawa P, Weinberg V, Fu KK (2002) Intensity-modulated radiotherapy in the treatment of nasopharyngeal carcinoma: an update of the UCSF experience. Int J Radiat Oncol Biol Phys 53: 12-22.

Liebig C, Ayala G, Wilks J, Verstovsek G, Liu H, Agarwal N, Berger DH, Albo D (2009) Perineural invasion is an independent predictor of outcome in colorectal cancer. J Clin Oncol 27: 5131-5137.

Liebig C, Ayala G, Wilks JA, Berger DH, Albo D (2009) Perineural invasion in cancer: a review of the literature. Cancer 115: 3379-3391.
Liu L, Liang S, Li L, Mao Y, Tang L, Tian L, Liao X, Cui C, Lin A, Ma J (2009) Prognostic impact of magnetic resonance imaging-detected cranial nerve involvement in nasopharyngeal carcinoma. Cancer 115: 1995-2003.

Lo YM, Chan AT, Chan LY, Leung SF, Lam CW, Huang DP, Johnson PJ (2000) Molecular prognostication of nasopharyngeal carcinoma by quantitative analysis of circulating Epstein-Barr virus DNA. Cancer Res 60: 6878-6881.

Miknyoczki SJ, Wan W, Chang H, Dobrzanski P, Ruggeri BA, Dionne CA, Buchkovich K (2002) The neurotrophin-trk receptor axes are critical for the growth and progression of human prostatic carcinoma and pancreatic ductal adenocarcinoma xenografts in nude mice. Clin Cancer Res 8: 1924-1931.

Ong CK, Chong VF (2010) Imaging of perineural spread in head and neck tumours. Cancer Imaging 10(Spec no. A): S92-S98.

Su CY, Lui CC (1996) Perineural invasion of the trigeminal nerve in patients with nasopharyngeal carcinoma. Imaging and clinical correlations. Cancer 78: 2063-2069.

Zhou GQ, Mao YP, Chen L, Li WF, Liu LZ, Sun Y, Chen Y, Tian L, Lin AH, Li L, Ma J (2012) Prognostic value of prevertebral space involvement in nasopharyngeal carcinoma based on intensity-modulated radiotherapy. Int J Radiat Oncol Biol Phys 82: 1090-1097.

Zhu Z, Friess H, DiMola FF, Zimmermann A, Graber HU, Korc M, Buchler MW (1999) Nerve growth factor expression correlates with perineural invasion and pain in human pancreatic cancer. J Clin Oncol 17: 2419-2428.

Zweig MH, Campbell G (1993) Receiver-operating characteristic (ROC) plots: a fundamental evaluation tool in clinical medicine. Clin Chem 39: 561-577.

This work is published under the standard license to publish agreement. After 12 months the work will become freely available and the license terms will switch to a Creative Commons AttributionNonCommercial-Share Alike 3.0 Unported License. 